\title{
Multivariate morphological brain signatures enable individualized prediction of dispositional need for closure
}

\author{
Xinling Chen ${ }^{1,2,3,4} \cdot$ Zhenhua $\mathrm{Xu}^{5,6,7} \cdot$ Ting $\mathrm{Li}^{8} \cdot$ Li Wang $^{9} \cdot$ Peiyi $\mathrm{Li}^{1,2,3,4} \cdot$ Han Xu $\mathrm{Xu}^{1,2,3,4} \cdot$ Chunliang Feng ${ }^{1,2,3,4,10}$. \\ Chao Liu ${ }^{5,6,7}$
}

Received: 22 March 2021 / Accepted: 28 September 2021 / Published online: 1 November 2021

(c) The Author(s), under exclusive licence to Springer Science+Business Media, LLC, part of Springer Nature 2021

\begin{abstract}
Need for closure (NFC) reflects stable individual differences in the desire for a quick, definite, and stable answer to a question. A large body of research has documented the association between NFC and various cognitive, emotional and social processes. Despite considerable interest in psychology, little effort has been made to uncover the neural substrates of individual variations in NFC. Herein, we took a data-driven approach to predict NFC trait combining machine learning framework and the whole-brain grey matter volume (GMV) features, which represent a reliable brain imaging measure and have been commonly employed to explore neural basis underlying individual differences of cognition and behaviors. Brain regions contributing to the prediction were then subjected to functional connectivity and decoding analyses for a quantitative inference on their psychophysiological functions. Our results indicated that multivariate patterns of GMV derived from multiple regions across distributed brain systems predicted NFC at individual level. The contributing regions are distributed across the emotional processing network (e.g., striatum), cognitive control network (e.g., lateral prefrontal cortex), social cognition network (e.g., temporoparietal junction) and perceptual processing network (e.g., occipital cortex). The current study provided the first evidence that dispositional NFC is embodied in multiple large-scale brain networks, helping to delineate a more complete picture about the neuropsychological processes that support individual differences in NFC. Beyond these findings, the current interdisciplinary approach to constructing and interpreting neuroimaging-based prediction model of personality traits would be informative to a wide range of future studies on personality.
\end{abstract}

Keywords Need for closure $\cdot$ Grey matter volume $\cdot$ Machine learning $\cdot$ Individual difference $\cdot$ Personality

Xinling Chen and Zhenhua Xu contributed equally to this work.

Chunliang Feng

chunliang.feng@m.scnu.edu.cn

$\triangle$ Chao Liu

liuchao@bnu.edu.cn

1 Key Laboratory of Brain, Cognition and Education Sciences (South China Normal University), Ministry of Education, Guangzhou, China

2 School of Psychology, South China Normal University, Guangzhou, China

3 Center for Studies of Psychological Application, South China Normal University, Guangzhou, China

4 Guangdong Key Laboratory of Mental Health and Cognitive Science, South China Normal University, Guangzhou, China

5 State Key Laboratory of Cognitive Neuroscience and Learning \& IDG/McGovern Institute for Brain Research, Beijing Normal University, 100875, Beijing, China
6 Center for Collaboration and Innovation in Brain and Learning Sciences, Beijing Normal University, 100875 Beijing, China

7 Beijing Key Laboratory of Brain Imaging and Connectomics, Beijing Normal University, 100875 Beijing, China

8 Institute of Brain Research and Rehabilitation (IBRR), South China Normal University, Guangzhou, China

9 Collaborative Innovation Center of Assessment for Basic Education Quality, Beijing Normal University, Beijing, China

10 Guangdong Provincial Key Laboratory of Mental Health and Cognitive Science, Center for Studies of Psychological Application, School of Psychology, South China Normal University, Guangzhou, China 


\section{Introduction}

We live in a world of abundant information, which provides a detailed reference for judgment, but also increases uncertainty in decision-making. Need for closure (NFC) is an important personality trait that affects knowledge construction in this regard (Krejci-Papa, 2010). Specifically, NFC represents stable individual differences in the desire for a quick, definite, and stable answer to a question and an intolerance of uncertainty (Kruglanski \& Fishman, 2009). The individual differences in the NFC could be reliably assessed with the Need for Closure Scale (NFCS) (Webster \& Kruglanski, 1994). People scoring high (vs. low) in the NFCS prefer order and predictability, are more closed-minded, decisive and aversive to ambiguity.

The past decades have witnessed a large body of research documenting the association between NFC and numerous intrapersonal and interpersonal phenomena, ranging from cognitive control (Kossowska, 2007b; Kossowska et al., 2010; Szumowska \& Kossowska, 2016) and emotional processing to economic decision making (Freeman et al., 2006), social cognition (Szumowska \& Kossowska, 2017) and social behaviors (Brizi \& Biraglia, 2020). First, NFC is linked to limitations in cognitive functioning (Szumowska \& Kossowska, 2016). High (vs. low) NFC individuals perform worse in working memory, and have lower capacity to employ controlled processing strategies (Kossowska et al., 2010). Notably, these cognitive process limitations might be compensated by efficient information selection associated with NFC, such that people scoring higher in the NFC are better at executive control tasks associated with selective attention (Kossowska et al., 2014; Szumowska \& Kossowska, 2017). Second, NFC is associated with levels of anxiety and depression among patients with mental disorders (Freeman et al., 2006), and intolerance of uncertainty as an inherent component of NFC constitutes a defining feature of anxiety disorders (Berenbaum et al., 2008; Starcevic $\&$ Berle, 2006). Third, NFC modulates a variety of decision making, such as internet use (Cudo et al., 2019) and food stockpiling during the COVID-19 pandemic (Brizi \& Biraglia, 2020). Likewise, laboratory evidence has indicated that individuals with a higher NFC exhibit higher willingness to choose smaller but certain or temporally more proximal options (Koscielniak et al., 2016; Schumpe et al., 2017). These findings implicate the influence of NFC on valuation processes, such as the intolerance of uncertainty (Berenbaum et al., 2008). Last, NFC has been associated with reduced perspective taking and empathetic concern toward dissimilar others (Baldner et al., 2020; Sparkman \& Blanchar, 2017). For instance, people high in NFC compared to those low in NFC are more likely to exhibit egocentric biases in a representative role (Stark \& Milyavsky, 2019). Accordingly, NFC has been proposed as a complicated construct related to a wide range of psychological processes (Roets et al., 2015).

Despite widespread interest in NFC in psychology, much less is known about the neural substrates underlying this personality trait. In the current work, we aimed to decode NFC from brain morphology with the purpose to reveal the structural anatomical correlates of NFC. In particular, we utilized a multivariate predictive approach under machine learning framework to predict individual level of NFC, based on brain structural features derived from high-resolution T1-weighted images. The novel data-driven approach employed in the current study provided a couple of advantages. First, the current multivariate approach allows for detecting subtle and spatially distributed associations of the NFC with morphological variations (Mur et al., 2009). This is particularly relevant for complicated psychological construct such as NFC, which has been previously implicated in diverse processes. Second, the machine learning approach typically implements cross-validation procedures to estimate the prediction model with training samples, and to test the model performance with independent dataset (i.e., test data), helping to establish robust relationship between individual differences in NFC and brain morphology (Chen et al., 2021; Cui et al., 2016; Feng, Cui, et al., 2019; Feng, Wang, et al., 2019; Feng et al., 2018; Feng et al., 2021; Feng et al., 2018).

Third, the current study employed the regional grey matter volume (GMV) derived from analysis of voxel-based morphometry (VBM) as predicting features, an automated technique for assessing structural variations in the brain. Notably, the VBM represents one of most reliable brain imaging measures (Zuo et al., 2019). Moreover, VBM has been commonly utilized in individual difference studies to examine neural substrates of the wide spectrum of human cognition and behaviors (Kanai \& Rees, 2011; Nash et al., 2015). This approach holds the assumption that individual differences in human brain morphometry (e.g., GMV) give rise to variation in personality traits and social preferences (Kanai \& Rees, 2011; Nash et al., 2015). Last, VBM is easy to use and has provided biologically plausible results (for a review, see also Whitwell, 2009). For these reasons, GMV represent a reasonable starting point to explore the neural basis of individual differences in NFC (Kanai \& Rees, 2011; Nash et al., 2015).

Finally, we implemented following-up network analysis and functional decoding analyses to characterize the psychophysiological functions of brain regions contributing to the prediction model. Specifically, the network analysis examined the network connectivity patterns (i.e., modules) among the contributing brain regions from a graph-theoretic framework, helping to interpret the identified brain regions from the network level rather than the regional level $(\mathrm{He}$ 
\& Evans, 2010; Xu et al., 2016). The functional decoding analysis allows for quantitative inference of the psychological functions of contributing modules based on broader neuroimaging literature from the Neurosynth database (Yarkoni et al., 2011).

In light of previous findings, it was hypothesized that brain systems implicated in cognitive control, emotional processing, valuation processes, and social cognitive functioning would be associated with individual variations in NFC. Accordingly, we expected that variations in morphology among these brain systems would contribute to predicting individual differences in NFC scores.

\section{Materials and methods}

\section{Participants}

One hundred and sixty-two healthy right-handed undergraduate or graduate students aged $18-36$ years (133 males; mean age \pm S.D.: $22.16 \pm 2.56$ years) were recruited for this study. The exclusion criteria included a history of neurological or psychiatric disorder. The current study did not collect information on whether participants had a prolonged use of a recreational or prescription drug. The study was conducted in accordance with the Declaration of Helsinki and approved by the Ethics Committee of Beijing Normal University. Written informed consent was obtained from all participants before the experiment.

\section{Need for closure scale}

A Chinese version of the need for closure scale (NFCS) was administered to evaluate individual differences in need for cognitive closure. The NFCS consists of 42 items rating on a 6-point Likert scale ranging from 1 (strong disagreement) to 6 (strong agreement) (Webster \& Kruglanski, 1994). Higher scores on the NFCS were interpreted as higher levels of need for cognitive closure. The discriminant validity and good reliability of NFCS have been reported in previous studies (Shiloh et al., 2001; Szumowska et al., 2018). In the current sample, the Cronbach's alpha coefficient of the NFCS is 0.71 , indicating that internal consistency reliability of the employed scale was acceptable.

\section{MRI data acquisition}

MRI images were acquired on a TRIO 3-Tesla Siemens scanner at the Imaging Center for Brain Research, Beijing Normal University. High-resolution structural images were acquired through a 3D sagittal T1-weighted magnetizationprepared rapid acquisition with gradient-echo (MPRAGE) sequence, adopting the following parameters: sagittal slices,
144; TR, $2530 \mathrm{~ms}$; TE, $3.39 \mathrm{~ms}$; slice thickness, $1.33 \mathrm{~mm}$; voxel size, $1 \times 1 \times 1.33 \mathrm{~mm}^{3}$; flip angle, $7^{\circ}$; inversion time, $1,100 \mathrm{~ms}$; and FOV, $256 \times 256 \mathrm{~mm}^{2}$.

In addition, all participants accomplished a resting-state fMRI scanning lasting for five minutes, during which they were instructed to close eyes, remain awake but not to think about anything deliberately during scanning. Following parameters were applied for the resting-state scanning: axial slices, 33; slice thickness, $3.5 \mathrm{~mm}$; gap, $0.7 \mathrm{~mm}$; TR, 2,000 $\mathrm{ms}$; TE, $30 \mathrm{~ms}$; flip angle, 90 ; voxel size, $3.5 \times 3.5 \times 3.5$ $\mathrm{mm}^{3}$; and FOV, $244 \times 244 \mathrm{~mm}^{2}$.

\section{Image preprocessing}

Each participant's grey matter volume (GMV) map was acquired by using the Computational Anatomy Toolbox (CAT12; http://dbm.neuro.uni-jena.de/cat/), implemented with Statistical Parametric Mapping software (SPM12; http://www.fil.ion.ucl.ac.uk/spm/) on Matlab platform. This processing procedure consisted of the following steps: (1) T1-weighted images of each participant were registered to the Montreal Neurological Institute (MNI) space; (2) the volumetric T1-weighted images were segmented into Grey Matter (GM), white matter (WM) and cerebrospinal fluid (CSF) density maps via the standard unified segmentation approach (Ashburner \& Friston, 2005); (3) the segmented GM density (GMD) map was spatially normalized to the International Consortium for Brain Mapping (ICBM) GM templates using the 12-parameter affine transformation (Ashburner et al., 1997), and nonlinear registration (Ashburner \& Friston, 1999); (4) the modulation was applied by multiplying the resulting GMD map with the nonlinear components of Jacobian determinant, which ensured the resulting GM volume (GMV) maps adjust for the resulting volume changes following the spatial normalization procedure (Good et al., 2001); and (5) GMV maps were smoothed using a 4-mm full-width at-half-maximum Gaussian kernel (FWHM). This FWHM value has been often employed in the multivariate predictive approach of neuroimaging data, by providing a balance between reserving anatomical details and compensating for registration errors (Cui et al., 2018; Franke et al., 2018; Hamann et al., 2014).

Resting-state imaging data were preprocessed using the Data Processing Assistant for Resting-State fMRI (DPARSF) (Yan et al., 2016; Yan \& Zang, 2010) based on SPM12. According to the recommended procedures (Yan et al., 2016; Yan \& Zang, 2010), before the formal processing, the first ten time points were eliminated to reduce the interference of signal equilibrium and allow participants' adaptation to scanning environment. Then slice timing was performed to correct acquisition time of each slice. Afterwards, images were realigned for correcting head movement. In particular, head movement was controlled by including 24 
movement regressors with autoregressive models of motion incorporating six head motion parameters, six head motion parameters one time point before and the 12 corresponding squared items (Friston et al., 1996). Seven participants (seven males) were excluded from further analysis under the criteria of head motion exceeding $2.0 \mathrm{~mm}$ maximum translation, $2.0^{\circ}$ rotation. Moreover, head motion was further controlled in the data preprocessing, such that volumes with an framewise displacement (FD) $>0.5 \mathrm{~mm}$, along with the immediately preceding volume and two subsequent volumes, were considered micromovement-containing volumes, and each of these volumes were modeled as a separate regressor in nuisance covariates regression (Power et al., 2014; Yan et al., 2013). In addition, functional images were normalized to Montreal Neurological Institute (MNI) space based on T1 images. Moreover, for the reduction of noise, the Gaussian smooth filter was used to reduce the impact of spatial noise $\left(\mathrm{FWHM}=6 \times 6 \times 6 \mathrm{~mm}^{3}\right)$. Lastly, in order to control the nuisance effects, the head motion parameter, global mean signal, the cerebrospinal fluid (CSF) and the white matter signals were regressed.

\section{Feature extraction}

Individual GMV maps were first partitioned into 120 regions of interest (ROIs) defined by the Automated Anatomical Labeling (AAL) atlas (Tzourio-Mazoyer et al., 2002). Afterwards, for each participant, the mean GMV value of each ROI was computed by averaging the GMV values of all voxels within a given ROI (Amico et al., 2011; Cui et al., 2016a). Accordingly, the mean GMV values of 120 ROIs were extracted as features of each participant for further analysis (Fig. 1).

\section{The multivariate Relevance Vector Regression (RVR) analysis}

The relationship between NFCS trait and brain morphometry was examined using multivariate RVR analysis implemented in the functions in PRoNTo (Schrouff et al., 2013) (http:// www.mlnl.cs.ucl.ac.uk/pronto/) under Matlab environment (Mathworks, 2016 release; and main function to call those subfunctions could be found in https://github.com/ZaixuCui/ Pattern_Regression_Clean). RVR has been demonstrated as an appropriate machine learning approach for complex information simulation with good robustness and acceptable computational efficiency (Wu et al., 2008). RVR is a pattern recognition method that uses a full probabilistic Bayesian inference to obtain sparse regression models. Sparsity is achieved in the classification of zero versus nonzero weights through the calculation of the Bayesian posterior distribution of all weights. In this process, the majority of weights peak at zero with relatively few nonzero weights, which are subsequently used to define parameter optimization. To constrain the maximum likelihood estimation of this model in this way, the weight distribution is applied with a zero-mean Gaussian prior probability distribution, which is controlled by a set of hyperparameters (Tipping, 2001). Specifically, the maximum possible values of these hyperparameters are estimated from the training data by using the iterative method. Moreover, training vectors with non-zero weights are expressed as correlation vectors for prediction or classification, given that the posterior distribution of most weights rapidly reaches sparsity near zero. Finally, an optimized posterior distribution is performed for the weights. By calculating the prediction distribution, the target value (NFC score) of the input value (120 GMV feature) can be predicted (Gong et al., 2014).

The performance of prediction was assessed by adopting a 10-fold cross-validation (Varoquaux et al., 2017). All participants were randomly divided into 10 subsets, of which 9 were used as the training set, and the remaining one was served as the testing set. A RVR prediction model was trained by the training set, hyper-parameters acquired from which was tested by the GMV features of testing data to predict NFCS scores. Then training and testing procedures were repeated 10 times, thus each subset was used as a testing set once. Since the full dataset was randomly divided into 10 subsets, performance might depend on the division of data. This issue was addressed by performing the 10-fold cross-validation 100 times, and the results were averaged to indicate the final prediction performance.

\section{Evaluation of prediction performance}

The accuracy of prediction was measured by two common statistics (Cui \& Gong, 2018; Cui et al., 2018; Franke et al., 2010; Gong et al., 2014): (i) the correlation coefficient (r); and (ii) mean absolute error (MAE). In the RVR analysis, $N$ predicted values were produced using the 10 -fold crossvalidation procedure for $N$ participants, based on which the correlation coefficient and $M A E$ between prediction scores and actual scores of NFCS could be calculated. To control for potential confounds of age, gender, and brain size (see also Table S1), the relationship between actual and predicted NFCS scores was calculated after excluding these confounding effects. Subsequently, the statistical significance of prediction accuracy (i.e., $r$ and $M A E$ ) was determined by a permutation test with 1,000 permutations to assess whether the obtained indices were significantly better than those expected by chance level. In each permutation, pairs of NFCS scores and GMV features were randomly permuted, and the 10-fold cross-validation approach was employed to predict the randomized targets. The number of times the permuted value was better than the true value was then divided by 1,000 providing an estimated $\mathrm{p}$-value for observed prediction accuracy. 


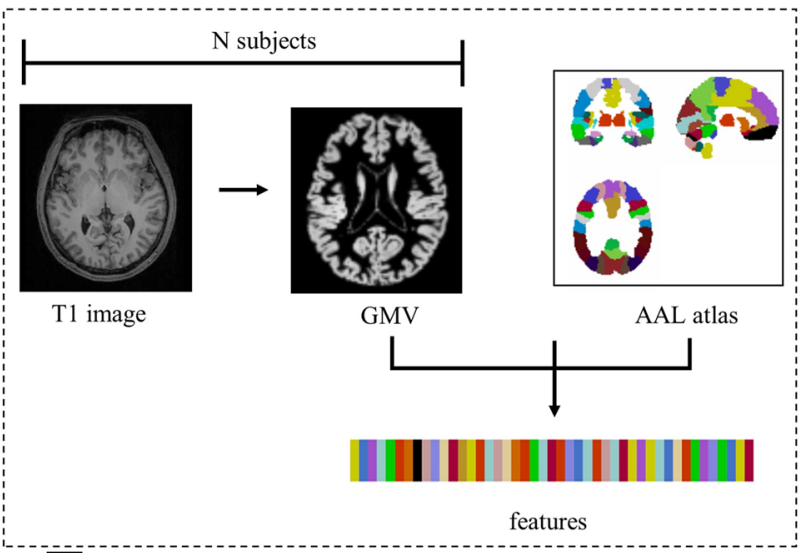

- Step 1: Feature extraction

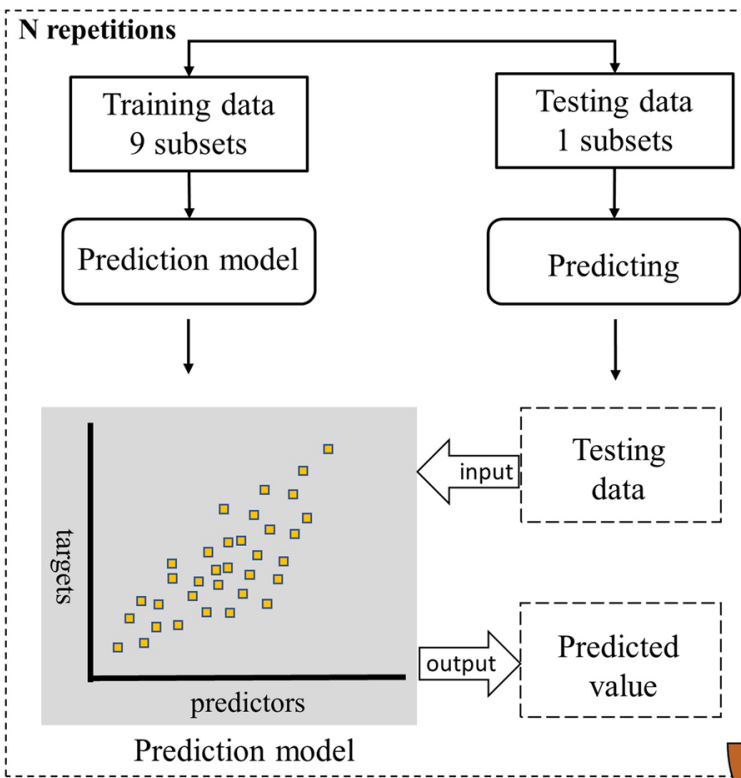

- Step 2: Model construction

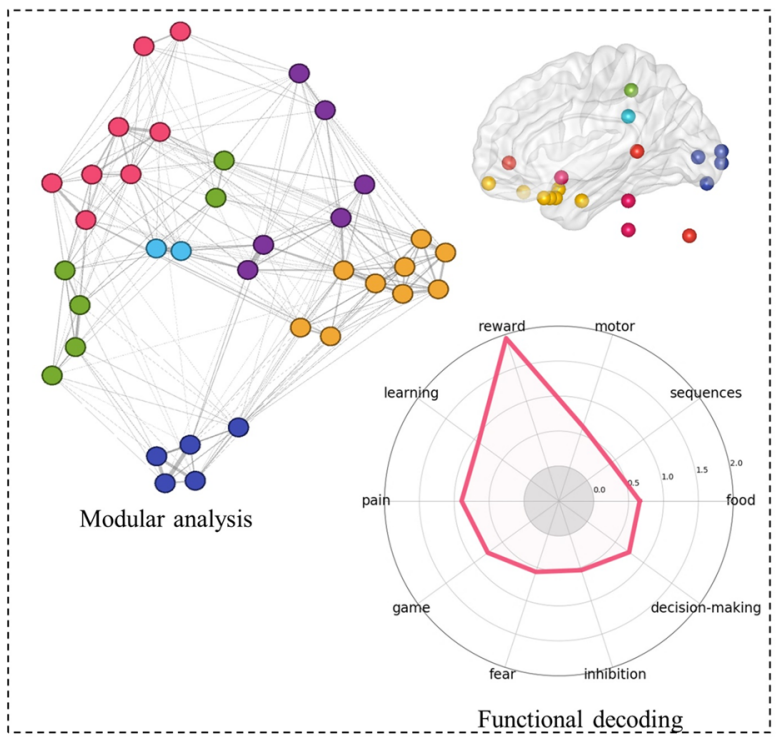

- Step 4: Model explanation

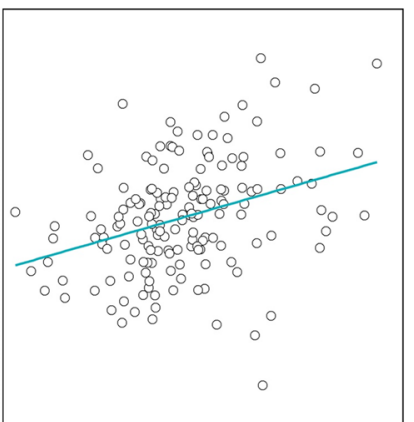

$r$

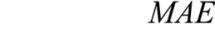

- Step 3: Model evaluation
Fig. 1 The prediction schematic flow using patterns of brain morphology. Step1 (Feature extraction): The mean GMV values of 120 ROIs defined by AAL were extracted as features of each participant. Step 2 (Model construction): The relationship between NFCS trait and brain morphometry was examined using multivariate RVR analysis, combining with the 10 -fold cross-validation to assess the prediction performance. Step 3 (Model evaluation): The accuracy of

\section{Contributing features in the prediction}

Since the absolute prediction weights of ROIs quantify the significance of GMV features in the prediction of NFCS scores, features with higher weights indicate potential contributing regions for the prediction (Cui \& Gong, 2018; Erus et al., 2015; Gong et al., 2014). Specifically, features with strong weights in the top $30 \%$ were selected for visualization and regarded as the predictive regions (Ecker et al., 2010; Gong et al., 2014). prediction was measured by the correlation coefficient $(r)$ and mean absolute error (MAE). Step 4 (Model explanation): The modular analysis and functional decoding analysis were conducted to reveal the neuropsychological functions of the contributing regions. GMV, grey matter volume; ROI, region of interest; AAL, Automated Anatomical Labeling; NFCS, the need for closure scale; RVR, the Relevance Vector Regression; $M A E$, mean absolute error

\section{Modular analysis}

To examine whether the contributing regions is intrinsically organized into functionally specific networks, we performed a modularity analysis using the Graph-theoretical Network Analysis Toolkit (Wang et al., 2015). Specifically, the mean time courses of all the voxels within each region of interest (ROI, i.e., brain regions with strong weights in the top 30\%) were extracted to calculate the Pearson correlation coefficient matrix 
for representing the resting brain functional network, resulting in a symmetric connectivity matrix for each participant. These matrices were Fisher $z$-transformed and averaged to obtain a mean matrix used for the following analyses (Xu et al., 2016).

To exclude the confounding impact of spurious relationships in internal connectivity matrices, the obtained mean matrix connectivity density value was set to range from 0.23 to 0.50 with a step length of 0.01 . The connectivity density value represents sparsity; that is, the ratio of the number of actual edges divided by the maximum possible number of edges (Bassett et al., 2008; He et al., 2009). For example, a connectivity density of 0.4 indicates that edges with strong connectivity strength in the top $40 \%$ were kept in the graph (Wang et al., 2015). The connectivity density value at the lower limit (i.e., 0.23) was set when the graph began to fragment into components and increased it by 0.01 until 0.5 to test the stability of results across various thresholds. Characterizing the graph across various thresholds provide more information than arbitrarily selecting a single threshold; therefore, the analysis scheme has been recommended in the literature (Rubinov \& Sporns, 2010; Wang et al., 2015). These low-value filtered matrices were performed for the modular analysis using the Graph-Theoretical Network Analysis Toolkit (Wang et al., 2015). The toolkit detects communities by maximizing the modularity $\mathrm{Q}$ with the spectral optimization algorithm, which has been introduced as a measure to assess the goodness of a partition (Newman, 2006; Newman \& Girvan, 2004). Finally, the number of modules and the membership of each ROI were obtained.

To validate the modularity partition derived from modular analyses, the resulting modules were overlaid onto seven canonical functional cortical networks, a collection of subcortical areas, and cerebellar regions (Choi et al., 2012; Liu et al., 2018; Yeo et al., 2011). Canonical networks include the fronto-parietal network, dorsal attention network, ventral attention network (i.e., salience network), somatomotor network, visual network, limbic network, and default mode network (Yeo et al., 2011). The relative distribution was calculated by the ratio of the number of contributing voxels of a given network versus the total number of all contributing voxels, while the absolute distribution was computed by the ratio of contributing voxels of a given network versus total number of voxels in the corresponding template network (Chen et al., 2018; Li et al., 2020; Zhang et al., 2017).

\section{Functional decoding for contributing modules}

To explore which psychological topics were most relevant to the identified modules, a meta-analysis was first performed based on the version 0.6 of the Neurosynth database (Yarkoni et al., 2011). The database consists of 11,406 fMRI studies and over 410,000 activity peaks that cover all-sided published neuroimaging literature. The observations for each study contain the peak activities for all contrasts reported in the study's table and the frequency of all words in the article abstract. Notably, a set of psychological 60 topics were used (De La Vega et al., 2018), which was derived by the latent Dirichlet allocation topic modeling to remedy the redundancy and potential ambiguity in word terms (Blei et al., 2003).

Using all fMRI studies, a functional decoding analysis was next performed by training a naïve Bayes classifier, which is widely used in text classification (Blei et al., 2003; Lewis, 1998). Two sets of studies that activated at least $5 \%$ voxels and that did not activate any voxel of the given region were selected respectively as the positive and negative samples of the training set (De La Vega et al., 2018). The area under the ROC curve (AUCROC) was used to measure the performance of the model with a 4-fold cross-validation. This resulted in the conditional probability of the 60 psychological topics under each module. Notably, only those topics that survived multiple comparisons using FDR with $P<0.01$ by implementing a permutation test were reported. Finally, the log odds ratio between the probability of a given topic activating the module was extracted from the trained naïve Bayes model to generate functional decoding profiles.

\section{Results}

\section{The relationship between GMV and NFCS scores}

Based on multivariate RVR and 10-fold cross-validation approach, the relationship between GMV and NFCS scores was assessed in a prediction model, which was then implemented to predict new data set. We found that the Pearson correlation coefficient $(r)$ and $M A E$ between predicted NFC scores and the actual scores were both significantly better than chance level $(r=0.26, P=0.001$, see Fig. $2 \mathrm{~A}$ and B; $M A E=$ $12.02, P=0.002$, see Fig. $2 \mathrm{C}$ and D).

\section{Contributing brain regions in the prediction of NFC}

ROIs with higher prediction weights in the top $30 \%$ were considered as predictive features for the prediction (Gong et al., 2014). To eliminate noise components and visualize the most predicting regions, 36 GMV features associated with higher prediction weights in the top $30 \%$ of the maximum weight value were listed (see Table 1 and Fig. 3).

\section{Modular analysis for contributing regions}

To detect the connectivity patterns between the identified contributing regions, we performed a modular analysis (i.e., a community detection algorithm). Five stable network modules were detected - default mode (DM) module, cortical affective (AF) module, central-executive (CE) 

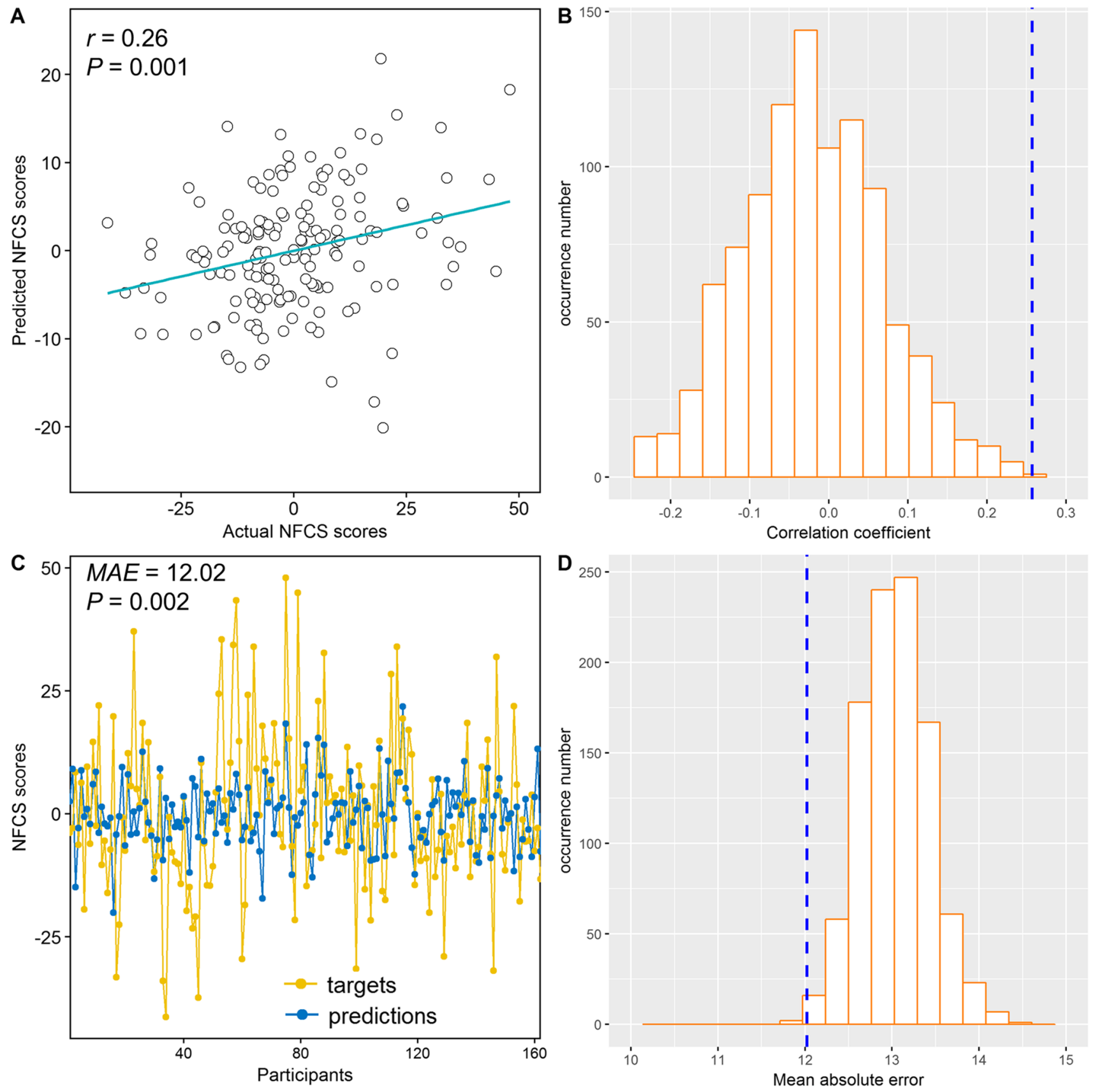

Fig. 2 Prediction performance based on GMV features. (A) The relationship between predicted NFCS scores and actual NFCS scores based on GMV features. (B) Permutation distribution of the prediction accuracy with the blue dashed line indicating the value obtained from real scores. (C) Consistency between prediction scores and

module (consisting of fronto-parietal network, somatomotor network and dorsal attention network), ventral attention (VA) module, visual module and subcortical (SC) module (Fig. 4D) - and the modules' partitioning maintained good consistency across different connectivity strengths (Fig. 4A). Specifically, the DM module mainly comprised medial superior frontal gyrus, posterior cingulate cortex (PCC), middle actual scores of NFCS (see also Fig. S1 for an enlarged version). (D) Permutation distribution of the MAE with blue dashed line indicating the value obtained from real scores. GMV, grey matter volume; NFCS, need for closure scale; MAE, mean absolute error

temporal gyrus and cerebellum posterior lobe. The AF module mainly consisted of olfactory cortex, medial orbitofrontal gyrus, gyrus rectus, orbitofrontal gyrus, hippocampus and parahippocampus. The CE module contained precentral gyrus, inferior frontal gyrus, supplementary motor area, postcentral gyrus, inferior parietal lobule and paracentral lobule. The VA module contained bilateral middle cingulate 
Table 1 Contributing regions

\begin{tabular}{|c|c|c|c|c|}
\hline Region & Hemisphere & MNI Coordinate & Module & Weights \\
\hline Medial superior frontal gyrus & $\mathrm{R}$ & $(18,44,-2)$ & $\mathrm{DM}$ & 0.15 \\
\hline Posterior cingulate gyrus & $\mathrm{L}$ & $(-16,-44,6)$ & $\mathrm{DM}$ & 0.10 \\
\hline Posterior cingulate gyrus & $\mathrm{R}$ & $(4,-44,6)$ & $\mathrm{DM}$ & 0.14 \\
\hline Middle temporal gyrus & $\mathrm{L}$ & $(-58,0,-34)$ & $\mathrm{DM}$ & 0.13 \\
\hline Cerebellum posterior lobe & $\mathrm{L}$ & $(-48,-56,-42)$ & $\mathrm{DM}$ & 0.19 \\
\hline Cerebellum posterior lobe & $\mathrm{R}$ & $(30,-80,-52)$ & $\mathrm{DM}$ & 0.12 \\
\hline Olfactory cortex & $\mathrm{R}$ & $(30,10,-20)$ & $\mathrm{AF}$ & 0.10 \\
\hline Medial orbitofrontal gyrus & $\mathrm{R}$ & $(10,58,-16)$ & $\mathrm{AF}$ & 0.16 \\
\hline Gyrus rectus & $\mathrm{R}$ & $(10,20,-26)$ & $\mathrm{AF}$ & 0.12 \\
\hline Medial orbitofrontal gyrus & $\mathrm{R}$ & $(14,16,-26)$ & $\mathrm{AF}$ & 0.11 \\
\hline Anterior orbitofrontal gyrus & $\mathrm{L}$ & $(-24,30,-22)$ & $\mathrm{AF}$ & 0.14 \\
\hline Anterior orbitofrontal gyrus & $\mathrm{R}$ & $(28,34,-22)$ & $\mathrm{AF}$ & 0.13 \\
\hline Posterior orbitofrontal gyrus & $\mathrm{R}$ & $(26,12,-26)$ & $\mathrm{AF}$ & 0.21 \\
\hline Hippocampus & $\mathrm{R}$ & $(28,-6,-28)$ & $\mathrm{AF}$ & 0.22 \\
\hline Parahippocampus & $\mathrm{L}$ & $(-20,-10,-36)$ & $\mathrm{AF}$ & 0.09 \\
\hline Precentral gyrus & $\mathrm{L}$ & $(-56,4,14)$ & $\mathrm{CE}$ & 0.12 \\
\hline Inferior frontal gyrus & $\mathrm{L}$ & $(-48,16,-2)$ & $\mathrm{CE}$ & 0.10 \\
\hline Supplementary motor area & $\mathrm{L}$ & $(0,6,44)$ & $\mathrm{CE}$ & 0.09 \\
\hline Postcentral gyrus & $\mathrm{L}$ & $(-64,-22,14)$ & $\mathrm{CE}$ & 0.20 \\
\hline Inferior parietal lobule & $\mathrm{L}$ & $(-56,-58,36)$ & $\mathrm{CE}$ & 0.17 \\
\hline Paracentral lobule & $\mathrm{R}$ & $(16,-40,48)$ & $\mathrm{CE}$ & 0.19 \\
\hline Middle cingulate gyrus & $\mathrm{L}$ & $(-12,-54,32)$ & VA & 0.15 \\
\hline Middle cingulate gyrus & $\mathrm{R}$ & $(12,-38,30)$ & VA & 0.10 \\
\hline Cuneus & $\mathrm{L}$ & $(-4,-102,12)$ & visual & 0.09 \\
\hline Cuneus & $\mathrm{R}$ & $(18,-102,6)$ & visual & 0.11 \\
\hline Superior occipital gyrus & $\mathrm{R}$ & $(24,-86,2)$ & visual & 0.14 \\
\hline Middle occipital gyrus & $\mathrm{R}$ & $(30,-102,-2)$ & visual & 0.10 \\
\hline Inferior occipital gyrus & $\mathrm{R}$ & $(34,-92,-16)$ & visual & 0.19 \\
\hline Caudate & $\mathrm{L}$ & $(-4,6,-12)$ & $\mathrm{SC}$ & 0.09 \\
\hline Caudate & $\mathrm{R}$ & $(14,8,-12)$ & $\mathrm{SC}$ & 0.09 \\
\hline Putamen & $\mathrm{L}$ & $(-24,0,-10)$ & $\mathrm{SC}$ & 0.13 \\
\hline Thalamus & $\mathrm{L}$ & $(-14,-30,-2)$ & $\mathrm{SC}$ & 0.20 \\
\hline Cerebellum anterior lobe & $\mathrm{L}$ & $(-8,-40,-28)$ & $\mathrm{SC}$ & 0.18 \\
\hline Cerebellum anterior lobe & $\mathrm{R}$ & $(16,-38,-28)$ & $\mathrm{SC}$ & 0.13 \\
\hline Cerebellum posterior lobe & $\mathrm{L}$ & $(-16,-36,-48)$ & $\mathrm{SC}$ & 0.10 \\
\hline Cerebellum posterior lobe & $\mathrm{R}$ & $(22,-38,-48)$ & $\mathrm{SC}$ & 0.30 \\
\hline
\end{tabular}

R, right; L, left. DM, default mode. AF, cortical affective. CE, central-executive. VA, ventral attention. SC, subcortical

gyrus. The visual module mainly comprised cuneus, superior occipital gyrus, middle occipital gyrus, and inferior occipital gyrus. The SC module mainly consisted of caudate, putamen, thalamus, cerebellum posterior lobe and cerebellum anterior lobe (Table 1).

For the connectivity density of 0.40 (a conventional value, e.g., Xu et al., 2016), a spring embedder layout model for straight-line representations was applied to group or separate nodes based on their connectivity patterns (Brandes \& Wagner, 1997). The spring-like formation of the three network modules was determined by the Euclidean distance between each pair of nodes (reflecting the graph-theoretic distance) and the thickness of lines (representing the connection strength of the edges) (Fig. 4B). The functional connectivity for ROIs sorted by modules demonstrated the strength of intra-module connections and inter-module connections, indicating that modules were clearly distinguished from each other (Fig. 4C).

Notably, the subnetworks derived from our modular analyses fit well with the canonical brain networks (Fig. 5A 
Fig. 3 Predictive regions. Modules from ROIs with top $30 \%$ higher prediction weights (DM module, purple; AF module, yellow; CE module, green; VA module, light blue; visual module, dark blue; SC module, pink). ROI, region of interest. DM, default mode. AF, cortical affective. CE, central-executive. VA, ventral attention. SC, subcortical

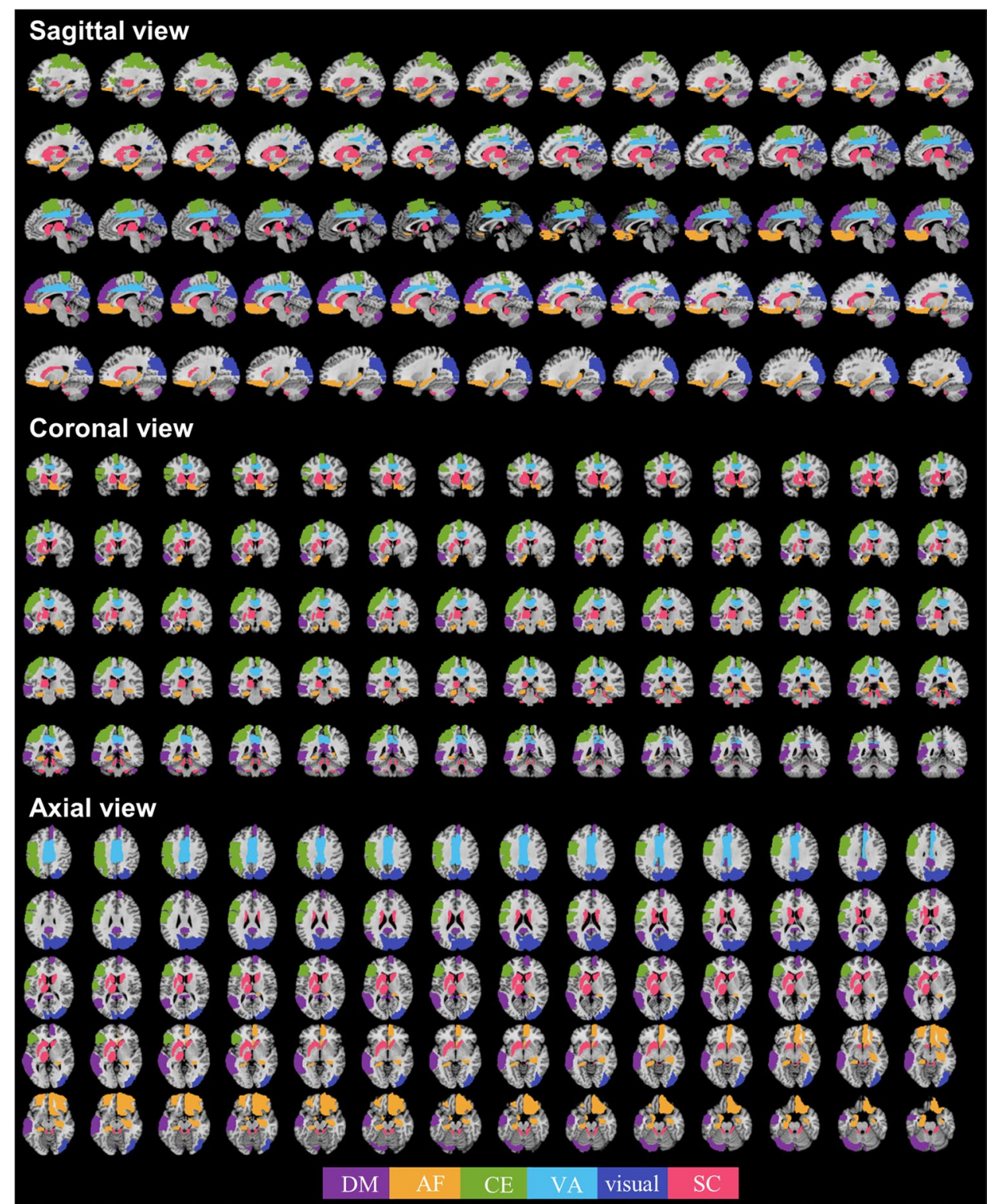

and B). To be specific, the DM module was primarily distributed in the default mode network (relative: $42.89 \%$; absolute: $15.92 \%$ ) and cerebellum (relative: $42.53 \%$; absolute: $19.00 \%$ ). The AF module was primarily distributed in the cortical affective network (relative: $36.82 \%$; absolute: $18.30 \%$ ). The CE module was primarily distributed in the somatomotor network (relative: $42.47 \%$; absolute: $26.76 \%$ ), fronto-parietal network (relative: $21.34 \%$; absolute: $13.41 \%$ ), and dorsal attention network (relative: $16.08 \%$; absolute: $12.91 \%$ ). The VA module was primarily distributed in the ventral attention network (relative: $45.26 \%$; absolute: $12.32 \%$ ). The visual module was primarily distributed in the visual network (relative: $84.13 \%$; absolute: $24.81 \%$ ). The SC module was primarily distributed in the subcortical network (relative: $85.88 \%$; absolute: $41.22 \%$ ).
Therefore, these results provided further validation of our modular analysis.

\section{Functional decoding for contributing modules}

Finally, we explored the modules' psychological functions by employing a data-driven approach to survey a broad range of fMRI studies in the Neurosynth database (Yarkoni et al., 2011) (Fig. 5C). The functional decoding analysis revealed that the DM module was predominantly associated with the psychological functions of reading, mentalizing, communication, emotion, awareness and memory. The AF module was mainly linked to fear, memory, emotion and learning. The CE module was linked to working memory, language, conflict and action. The VA module was associated with pain, conflict and decision-making. The 


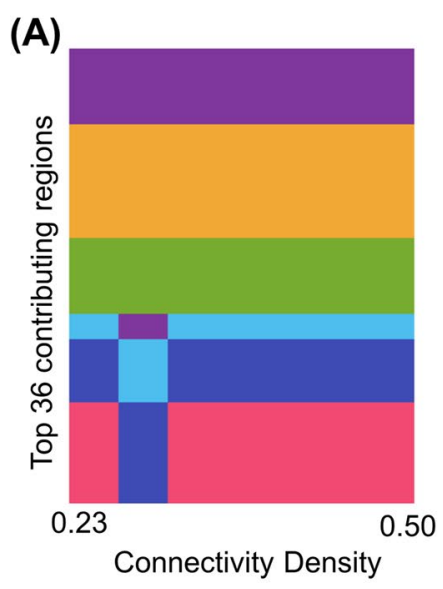

(B)

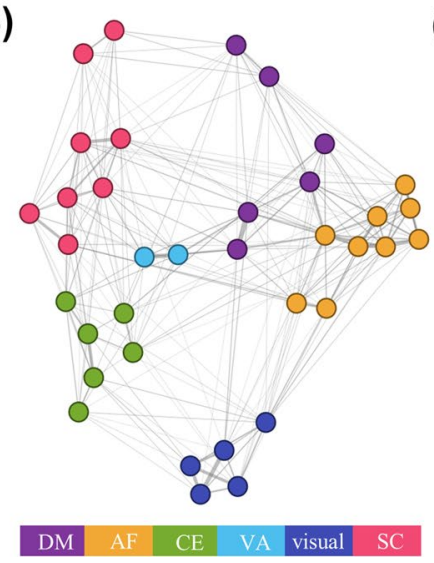

(C)

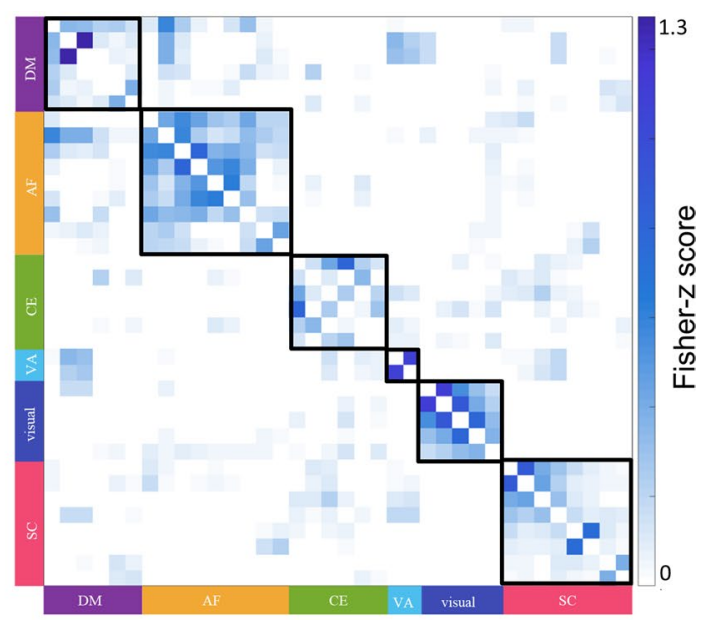

(D)
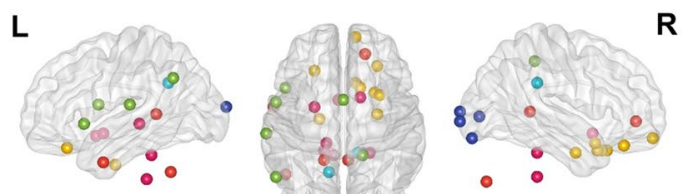

$\mathbf{R}$
Fig. 4 Psychophysiological functions of brain regions contributing to the GMV-based prediction model of NFC. (A) The modular analysis determined six stable modules from ROIs shown in the same color under connectivity density levels ranging from 0.23 to 0.50 by increments of 0.01. (B) The spring-like layout of the six network modules for a connectivity density of 0.40 displays the Euclidean distance between each pair of nodes. The thickness of lines indicates the connection strength of the edges. (C) Functional connectivity matrix for a connectivity density of 0.40 (ROIs are sorted by modules) showing the strength of edges within and those between modules. (D) The GMV-based prediction model determined 36 contributing regions (i.e., ROIs). ROIs outside of the template are cerebellar regions, given that the template does not contain the cerebellum. The colors indicate different brain network modules. GMV, grey matter volume. DM, default mode. AF, cortical affective. CE, central-executive. VA, ventral attention. SC, subcortical. R, right; L, left visual module was associated with visual-motion, attention, spatial, face/emotion and communication. The SC module was mainly linked to reward, decision-making, learning and pain.

\section{Discussion}

NFC has received widespread attention in the field of psychology over the past three decades, with well-documented relationship between NFC and a wide range of individual, interpersonal and group behaviors (Flynn et al., 2010; Marchlewska et al., 2018). Despite considerable investigation on the association between NFC and various behaviors and psychological processes, little effort has been made to examine the neural substrates of NFC (Kossowska et al., 2019; Viola et al., 2014). To address this issue, we first conducted individualized prediction of NFC trait under machine learning framework based on the whole-brain GMV features, with the purpose of exploring the neurobiological underpinnings of NFC. Next, we conducted modular detection to quantitatively characterize topological properties of the brain network constructed from resting-state functional connectivity of brain regions contributing to the prediction of NFC. Lastly, we performed a functional decoding analysis for modules contributing to NFC prediction for quantitative inference of psychological functions based on broader neuroimaging literature from the Neurosynth database (Yarkoni et al., 2011). Our results indicated that multivariate patterns of GMV derived from multiple regions across distributed brain systems predicted individual NFC propensity. Specifically, the individual differences of NFC were predicted by GMV features across hippocampus, caudate, 
pallidum, ventrolateral prefrontal cortex (vlPFC), supplementary motor area (SMA), dorsolateral prefrontal cortex (dIPFC), posterior cingulate cortex (PCC), temporoparietal junction (TPJ) and subregions of the cerebellum. Additionally, our modular and functional decoding analyses revealed that the brain regions contributing to the prediction model are mapped onto six large-scale network modules including the DM, AF, VA, SCN, CE and visual modules, which are implicated in social cognition, emotional processing, cognitive control, and perception.

First, the DM module mainly comprises vmPFC, PCC, dmPFC and TPJ, overlaps with canonical default mode network and is associated with mentalizing and communications as indicated by functional decoding analysis. The current results on the link between DM module and NFC are in line with previous findings that NFC plays a key role in social cognitive processes, including perspectivetaking, empathy and interpersonal communications (Flynn et al., 2010). For instance, individuals with higher NFC are less likely to accept a dissimilar target's response as appropriate and report less empathic concerns for the target (Baldner et al., 2020; Nelson et al., 2003). In addition, people high in NFC compared with those low in NFC spend less amount of effort to search for common ground during interpersonal communication, resulting in excessive biases in the direction of their own perspectives (Richter \& Kruglanski, 1999). Lastly, a high NFC enhances self-serving bias in both judicial predictions and fair value assessments among law-school students taking a representative role (Stark \& Milyavsky, 2019). In general, it requires a lot of effort to take the perspective of others, but NFC might induce reductions in the readiness to put mental effort into the processing of information, leading to attenuated perspective taking and empathic concern of dissimilar others (Kruglanski \& Chun, 2008).

Second, NFC is linked to morphological variations in brain networks important in affective processing, including the AF module (mainly consisting of OFC and hippocampus), SC module (mainly consisting of caudate, putamen and cerebellum) and VA module (mainly consisting of MCC). These modules mainly comprise regions in the striatal-cortical pathway and limbic/paralimbic system that are important in valuation processes and aversive emotion processing, as revealed by the functional decoding analysis. These findings complement several lines of evidence reported in the current literature. First, NFC has been found to modulate economic decision-making closely related to valuation processes, such that individuals higher in NFC are more risk-aversive and exhibit greater discounting of delayed rewards (Koscielniak et al., 2016; Schumpe et al., 2017). Second, NFC scales with emotional distress in both general populations (Berenbaum et al., 2008) and clinical populations with anxiety disorders (Freeman et al., 2006). For instance, self-reports of distress feelings, as well as cardiovascular measures (e.g., systolic blood pressure and heart rate), are increased in proportion with dispositional NFC in the decision-making processes (Wronska et al., 2019). Similarly, individuals scoring higher in the NFC experience more negative affect when they encounter an inconsistent (vs. consistent) cognition, regardless of whether the ultimate outcome is positive or negative (Di Santo et al., 2020; Wronska et al., 2019). Lastly, intolerance of uncertainty, as a key element of NFC (Kossowska et al., 2019), represents a key risky or maintaining factor of anxiety disorders (Starcevic \& Berle, 2006). In short, NFC as an epistemic motivation may induce negative affect when closure is threatened or undermined and positive affect when it is facilitated or attained, suggesting a close relationship between NFC and emotional processing.

Third, the CE module is embedded in canonical networks of fronto-parietal network, somatomotor network and dorsal attention network, consists of precentral gyrus, inferior frontal gyrus, supplementary motor area, postcentral gyrus, inferior parietal lobule and middle cingulate gyrus, brain regions playing a critical role in high-level cognitive control as indicated by functional decoding. In line with current findings, both behavioral and neural studies have demonstrated that NFC is related to cognitive control processes (Kossowska et al., 2019; Roets et al., 2015; Szumowska \& Kossowska, 2016, 2017; Todor, 2014). On the one hand, there is evidence showing that NFC is positively related to slower performance rate in a short-term memory task, lower effectiveness of cognitive control, and worse performance in a simple attentional task (Kossowska, 2007a, 2007b; Roets \& Hiel, 2008). A recent neuroimaging study has reported that the attenuated online adjustment of cognitive control among individuals high in NFC is associated with attenuated functional connectivity between dlPFC and vlPFC (Viola et al., 2014), both of which play a key role in behavioral adaptation (Thompson-Schill et al., 2002). Moreover, individuals high (vs. low) in NFC exhibit attenuated sensitivity to cues for response adjustment and decreased sensitivity to committing errors, manifesting as lower N2 response to stimulus-response congruency and smaller ERN responses, respectively (Kossowska, Czarnek, Wronka, Wyczesany, \& Bukowski, 2014). Similarly, decisiveness as a key facet of NFC is linked to worse performance in the stop-signal tasks, in parallel with lower N1 and $\mathrm{P} 3$ responses to stop signals as well as decreased ERN amplitudes to error responses (Senderecka et al., 2018). On the other hand, a growing body of evidence has indicated that high (vs. low) NFC individuals perform better in the tasks involving selective attention, suggesting that NFC is associated with efficient selection of relevant information from the environment (Kossowska, 2007a, 2007b). This idea is supported by a recent eye-tracking study showing that individuals with high (vs. low) NFC have a greater 
(A)
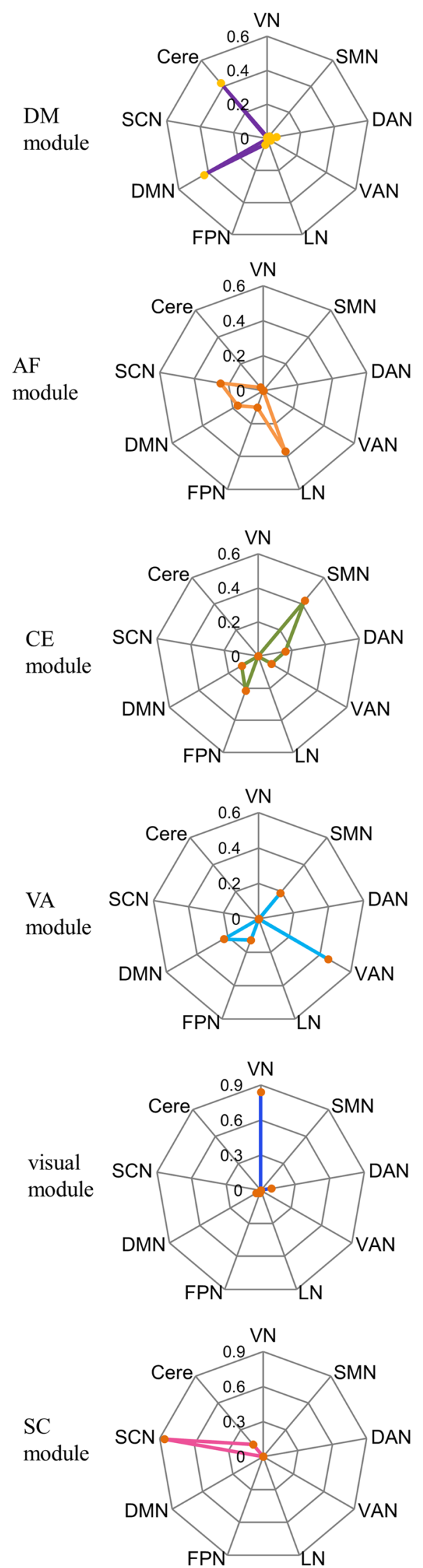

(B)
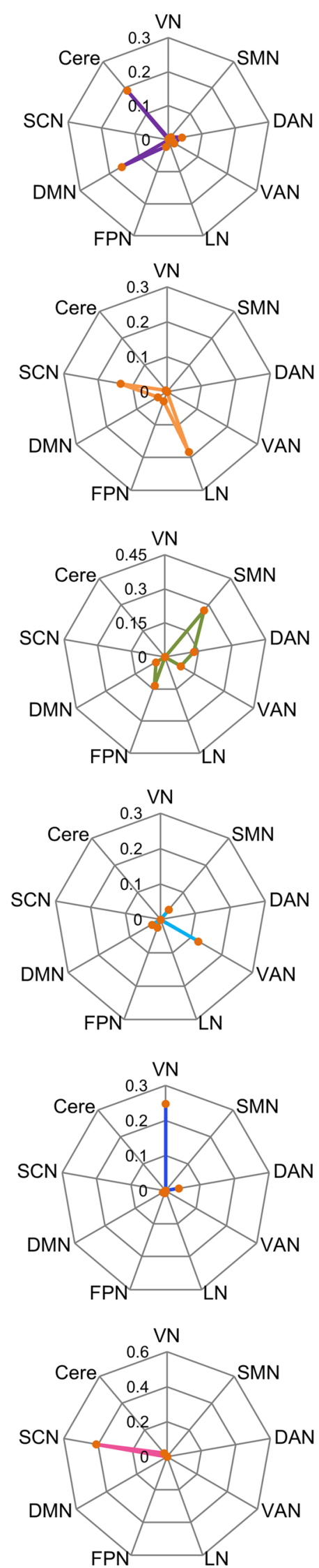

(C)
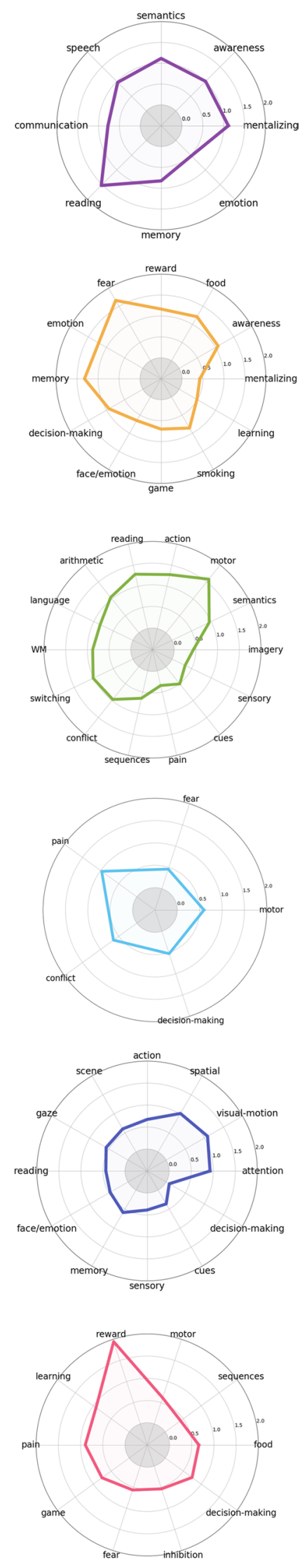
४Fig.5 Quantitative functional profiling of identified networks. (A) Relative distribution of DM, AF, CE, VA, visual and SC modules in canonical brain networks including VN, SCN, DMN, FPN, LN, VAN, DAN and SMN. (B) Absolute distribution of DM, AF, CE, VA, visual and SC modules in canonical brain networks. (C) Functional profiling of DM, AF, CE, VA, visual and SC modules. The log odds ratio between the probability of a given topic activating the network was displayed in a functional decoding profile for each network. DMN, default mode network; VN, visual network; SCN, subcortical network; FPN, fronto-parietal network; LN, limbic network; VAN, ventral attention network; DAN, dorsal attention network; SMN, somatomotor network; Cere, cerebellum

focus of attention (Szumowska \& Kossowska, 2017). Taken together, NFC might be better viewed as a goal, whose particular cognitive and behavioral consequences depend on the perceived means to that goal's pursuit, instead of cognitive deficit (Roets et al., 2015).

Lastly, the visual module includes occipital regions, which are primarily located in the canonical visual network and implicated in perceptual processing. In line with current results, there is evidence showing the association between NFC and perceptual decision-making, such that NFC is associated with urgency in perceptual decision-making (Evans, Rae, Bushmakin, Rubin, \& Brown, 2017). Moreover, NFC interacts with context to impact perceptual decision making, such that low NFC individuals' performance depends on task difficulty but not outcome relevance, whereas high NFC individuals' performance depends on outcome relevance but not task difficulty (Viola et al., 2015). Nevertheless, it should be noted that the effects of NFC on perceptual decisions are primarily attributed to the high-level strategies in decision making, whereas the current findings provide the first evidence to indicate that NFC might modulate low-level perceptual processes.

Several limitations related to the current study should be noted. First, we focused on the dispositional NFC in this study. However, NFC could also represent a transient state induced by conditional determinants (Roets et al., 2015). This conditional NFC, similar to trait NFC, has impact on a variety of cognition processing and human behaviors (Webber et al., 2018). Therefore, future studies are awaited to examine the common and/or distinct neural signatures between dispositional NFC and conditional NFC. Likewise, the current study examined the neural signatures underlying NFC as a whole, while previous studies have revealed different factors of NFC (Webster \& Kruglanski, 1994). Therefore, future studies are needed to examine neural substrates of different aspects of NFC. Second, it should be noted that predicted scores exhibited smaller variance than the actual scores, which could be attributed to regression prediction models shrinking estimates toward the mean, as well as the fact that the current predictive model did not fit perfectly with the actual scores. In this regard, future studies are encouraged to combine other neuroimaging measures (e.g., functional properties) to achieve a better and reliable neuroimaging-based prediction model of NFC. Moreover, the current findings were revealed in a single group and future studies are required to test the current prediction model in an independent sample (Masouleh et al., 2019). Third, the current study examined the structural anatomical correlates of NFC, and it remains unclear how the structural variations associated with NFC give rise to neuropsychological functioning associated with NFC. In this regard, our explanations on the functions of brain networks contributing to the prediction of NFC should be considered as tentative, although they are in line with a large body of evidence in the psychological field. Lastly, only a small group of females was included in the current study, and future studies are needed to recruit comparable male and female participants to examine potential gender differences.

Despite the limitations, the current research provided the first evidence that GMV of multiple brain systems enabled prediction of NFC scores at individual level. This might contribute to re-recognizing NFC fundamentally because there has been little evidence on the biological basis of NFC. Moreover, the interdisciplinary approach employed in the current study to constructing and interpreting neuroimaging-based prediction model of personality traits would be informative to a wide range of future studies on personality.

\section{Conclusions}

Taken together, the current study revealed that NFC is associated with morphological variations in multiple brain networks important for emotion processing, social cognition, cognitive control and perceptual processing. The current findings are consistent with a variety of neuropsychological processes implicated in previous studies, supplementing a view of neural mechanism of previous behavioral findings associated with NFC. Moreover, the current work also offers two novel perspectives for better understanding of NFC. First, the current multivariate approach helps to reveal the role of NFC in multiple psychological processes in a single study, helping to delineate a more complete picture of NFC. Second, the current findings also implicate the effects of NFC on psychological functions (e.g., perception) that have been much less studied, providing new insights for future research.

Supplementary Information The online version contains supplementary material available at https://doi.org/10.1007/s11682-021-00574-w.

Author contributions C.F. and C.L. conceived and designed the experiment; C.F., Z.X., T.L. and L.W. analyzed the data. C.F., X.C. and Z.X. interpreted the results and wrote the paper; C.F., X.C., P.L. and H.X. revised the paper. 
Funding This study was supported by the National Natural Science Foundation of China (31900757, 32020103008, 31871094, 32130045), the Natural Science Foundation of Guangdong Province (2021A1515010746), the Major Project of National Social Science Foundation (19ZDA363), and the Beijing Municipal Science and Technology Commission (Z151100003915122).

Data Availability Data and code are available upon reasonable request to the corresponding author.

\section{Declarations}

Ethical approval The study was conducted in accordance with the Declaration of Helsinki and approved by the Ethics Committee of Beijing Normal University.

Consent to participate Written informed consent was obtained from all participants before the experiment.

Consent to publish Authors have read and approved the final version of the manuscript.

Conflict of interest statement The authors declare that there is no conflict of interest.

\section{References}

Amico, F., Stauber, J., Koutsouleris, N., \& Frodl, T. (2011). Anterior cingulate cortex gray matter abnormalities in adults with attention deficit hyperactivity disorder: a voxel-based morphometry study. Psychiatry Research: Neuroimaging, 191(1), 31-35.

Ashburner, J., \& Friston, K. J. (1999). Nonlinear spatial normalization using basis functions. Human Brain Mapping, 7(4), 254-266.

Ashburner, J., \& Friston, K. J. (2005). Unified segmentation. NeuroImage, 26(3), 839-851.

Ashburner, J., Neelin, P., Collins, D., Evans, A., \& Friston, K. (1997). Incorporating prior knowledge into image registration. NeuroImage, 6(4), 344-352.

Baldner, C., Di Santo, D., Talamo, A., \& Pierro, A. (2020). Sympathy as knowledge of the other in need: An investigation into the roles of need for closure and the moral foundations on sympathy toward immigrants. Journal of Applied Social Psychology, 50(4), 240-252.

Bassett, D. S., Bullmore, E., Verchinski, B. A., Mattay, V. S., Weinberger, D. R., \& Meyer-Lindenberg, A. (2008). Hierarchical organization of human cortical networks in health and schizophrenia. Journal of Neuroscience, 28(37), 9239-9248.

Berenbaum, H., Bredemeier, K., \& Thompson, R. J. (2008). Intolerance of uncertainty: Exploring its dimensionality and associations with need for cognitive closure, psychopathology, and personality. Journal of Anxiety Disorders, 22(1), 117-125.

Blei, D. M., Ng, A. Y., \& Jordan, M. I. (2003). Latent dirichlet allocation. Journal of Machine Learning Research, 3(Jan), 993-1022.

Brandes, U., \& Wagner, D. (1997). A Bayesian paradigm for dynamic graph layout. Paper presented at the International Symposium on Graph Drawing.

Brizi, A., \& Biraglia, A. (2020). "Do I have enough food?" How need for cognitive closure and gender impact stockpiling and food waste during the COVID-19 pandemic: A cross-national study in India and the United States of America. Personality and Individual Differences, 168, 110396.
Chen, T., Becker, B., Camilleri, J., Wang, L., Yu, S., Eickhoff, S. B., \& Feng, C. (2018). A domain-general brain network underlying emotional and cognitive interference processing: evidence from coordinate-based and functional connectivity meta-analyses. Brain Structure and Function, 223(8), 3813-3840.

Chen, X., Xu, Y., Li, B., Wu, X., Li, T., Wang, L., \& Feng, C. (2021). Intranasal vasopressin modulates resting state brain activity across multiple neural systems: Evidence from a brain imaging machine learning study. Neuropharmacology, 190, 108561.

Choi, E. Y., Yeo, B. T., \& Buckner, R. L. (2012). The organization of the human striatum estimated by intrinsic functional connectivity. Journal of neurophysiology, 108(8), 2242-2263.

Cudo, A., Kopiś, N., Jarząbek-Cudo, A., Szewczyk, R., \& Demczuk, M. (2019). The relationship between problematic internet use and the need for cognitive closure. Advances in Psychiatry and Neurology/Postępy Psychiatrii i Neurologii, 28(3), 199-207.

Cui, Z., \& Gong, G. (2018). The effect of machine learning regression algorithms and sample size on individualized behavioral prediction with functional connectivity features. NeuroImage, $178,622-637$.

Cui, Z., Su, M., Li, L., Shu, H., \& Gong, G. (2018). Individualized prediction of reading comprehension ability using gray matter volume. Cerebral Cortex, 28(5), 1656-1672.

Cui, Z., Xia, Z., Su, M., Shu, H., \& Gong, G. (2016). Disrupted white matter connectivity underlying developmental dyslexia: a machine learning approach. Human Brain Mapping, 37(4), $1443-1458$.

De La Vega, A., Yarkoni, T., Wager, T. D., \& Banich, M. T. (2018). Large-scale meta-analysis suggests low regional modularity in lateral frontal cortex. Cerebral Cortex, 28(10), 3414-3428.

Di Santo, D., Chernikova, M., Kruglanski, A. W., \& Pierro, A. (2020). Does inconsistency always lead to negative affect? The influence of need for closure on affective reactions to cognitive inconsistency. International Journal of Psychology, 55(5), 882-890.

Ecker, C., Marquand, A., Mourão-Miranda, J., Johnston, P., Daly, E. M., Brammer, M. J., \& Williams, S. C. (2010). Describing the brain in autism in five dimensions - magnetic resonance imagingassisted diagnosis of autism spectrum disorder using a multiparameter classification approach. Journal of Neuroscience, 30(32), 10612-10623.

Erus, G., Battapady, H., Satterthwaite, T. D., Hakonarson, H., Gur, R. E., Davatzikos, C., \& Gur, R. C. (2015). Imaging patterns of brain development and their relationship to cognition. Cerebral Cortex, 25(6), 1676-1684.

Evans, N. J., Rae, B., Bushmakin, M., Rubin, M., \& Brown, S. D. (2017). Need for closure is associated with urgency in perceptual decision-making. Memory \& Cognition, 45(7), 1193-1205.

Feng, C., Cui, Z., Cheng, D., Xu, R., \& Gu, R. (2019). Individualized prediction of dispositional worry using white matter connectivity. Psychological Medicine, 49(12), 1999-2008.

Feng, C., Wang, L., Li, T., \& Xu, P. (2019). Connectome-based individualized prediction of loneliness. Social Cognitive and Affective Neuroscience, 14(4), 353-365.

Feng, C., Yuan, J., Geng, H., Gu, R., Zhou, H., Wu, X., \& Luo, Y. (2018). Individualized prediction of trait narcissism from wholebrain resting-state functional connectivity. Human Brain Mapping, 39(9), 3701-3712.

Feng, C., Zhu, Z., Cui, Z., Ushakov, V., Dreher, J. C., Luo, W., \& Krueger, F. (2021). Prediction of trust propensity from intrinsic brain morphology and functional connectome. Human Brain Mapping, 42(1), 175-191

Feng, C., Zhu, Z., Gu, R., Wu, X., Luo, Y. J., \& Krueger, F. (2018). Resting-state functional connectivity underlying costly punishment: a machine-learning approach. Neuroscience, 385, 25-37. 
Flynn, F. J., Reagans, R. E., \& Guillory, L. (2010). Do you two know each other? Transitivity, homophily, and the need for (network) closure. Journal of Personality and Social Psychology, 99(5), 855.

Franke, K., Gaser, C., Roseboom, T. J., Schwab, M., \& de Rooij, S. R. (2018). Premature brain aging in humans exposed to maternal nutrient restriction during early gestation. NeuroImage, 173, 460-471.

Franke, K., Ziegler, G., Klöppel, S., Gaser, C., \& Initiative, A. s. D. N. (2010). Estimating the age of healthy subjects from T1-weighted MRI scans using kernel methods: exploring the influence of various parameters.Neuroimage, 50(3), 883-892.

Freeman, D., Garety, P., Kuipers, E., Colbert, S., Jolley, S., Fowler, D., \& Bebbington, P. (2006). Delusions and decision-making style: use of the Need for Closure Scale. Behaviour Research and Therapy, 44(8), 1147-1158.

Friston, K. J., Williams, S., Howard, R., Frackowiak, R. S., \& Turner, R. (1996). Movement-related effects in fMRI time-series. Magnetic Resonance in Medicine, 35(3), 346-355.

Gong, Q., Li, L., Du, M., Pettersson-Yeo, W., Crossley, N., Yang, X., $\&$ Mechelli, A. (2014). Quantitative prediction of individual psychopathology in trauma survivors using resting-state FMRI. Neuropsychopharmacology, 39(3), 681-687.

Good, C. D., Johnsrude, I., Ashburner, J., Henson, R. N., Friston, K. J., \& Frackowiak, R. S. (2001). Cerebral asymmetry and the effects of sex and handedness on brain structure: a voxel-based morphometric analysis of 465 normal adult human brains. NeuroImage, 14(3), 685-700.

Hamann, J. M., Dayan, E., Hummel, F. C., \& Cohen, L. G. (2014). Baseline frontostriatal-limbic connectivity predicts reward-based memory formation. Human Brain Mapping, 35(12), 5921-5931.

He, Y., Dagher, A., Chen, Z., Charil, A., Zijdenbos, A., Worsley, K., \& Evans, A. (2009). Impaired small-world efficiency in structural cortical networks in multiple sclerosis associated with white matter lesion load. Brain, 132(12), 3366-3379.

He, Y., \& Evans, A. (2010). Graph theoretical modeling of brain connectivity. Current Opinion in Neurology, 23(4), 341-350.

Kanai, R., \& Rees, G. (2011). The structural basis of inter-individual differences in human behaviour and cognition. Nature Reviews Neuroscience, 12(4), 231-242.

Koscielniak, M., Rydzewska, K., \& Sedek, G. (2016). Effects of age and initial risk perception on balloon analog risk task: The mediating role of processing speed and need for cognitive closure. Frontiers in Psychology, 7, 659.

Kossowska, M. (2007). Motivation towards closure and cognitive processes: An individual differences approach. Personality and Individual Differences, 43(8), 2149-2158.

Kossowska, M. (2007). The role of cognitive inhibition in motivation toward closure. Personality and Individual Differences, 42(6), $1117-1126$.

Kossowska, M., Bukowski, M., \& Czarnek, G. (2014). Two routes to closure: Time pressure and goal activation effects on executive control. Polish Psychological Bulletin, 45(3), 268-274.

Kossowska, M., Czarnek, G., Wronka, E., Wyczesany, M., \& Bukowski, M. (2014). Individual differences in epistemic motivation and brain conflict monitoring activity. Neuroscience Letters, 570, 38-41.

Kossowska, M., Orehek, E., \& Kruglanski, A. W. (2010). Motivation towards closure and cognitive resources: An individual differences approach. In Handbook of individual differences in cognition (pp. 369-382). Springer

Kossowska, M., Szwed, P., \& Wyczesany, M. (2019). Motivational effects on brain activity: need for closure moderates the impact of task uncertainty on engagement-related P3b. NeuroReport, 30(17), 1179-1183.

Krejci-Papa, M. (2010). Need for cognitive closure and information search strategy. Psychology \& Marketing, 25(11), 1027-1042.
Kruglanski, A. W., \& Chun, W. Y. (2008). Motivated closed-mindedness and its social consequences. Handbook of motivation science, 84-99.

Kruglanski, A. W., \& Fishman, S. (2009). The need for cognitive closure. Handbook of individual differences in social behavior, 343-353.

Lewis, D. D. (1998). Naive (Bayes) at forty: The independence assumption in information retrieval. Paper presented at the European conference on machine learning.

Li, T., Wang, L., Camilleri, J. A., Chen, X., Li, S., Stewart, J. L., \& Feng, C. (2020). Mapping common grey matter volume deviation across child and adolescent psychiatric disorders. Neuroscience \& Biobehavioral Reviews, 115, 273-284.

Liu, J., Liao, X., Xia, M., \& He, Y. (2018). Chronnectome fingerprinting: Identifying individuals and predicting higher cognitive functions using dynamic brain connectivity patterns. Human Brain Mapping, 39(2), 902-915.

Marchlewska, M., Cichocka, A., \& Kossowska, M. (2018). Addicted to answers: Need for cognitive closure and the endorsement of conspiracy beliefs. European Journal of Social Psychology, 48(2), 109-117.

Masouleh, S. K., Eickhoff, S. B., Hoffstaedter, F., Genon, S., \& Initiative, A. (2019). Empirical examination of the replicability of associations between brain structure and psychological variables. eLife, 8, e43464.

Mur, M., Bandettini, P. A., \& Kriegeskorte, N. (2009). Revealing representational content with pattern-information fMRI - an introductory guide. Social Cognitive and Affective Neuroscience, 4(1), 101-109.

Nash, K., Gianotti, L. R., \& Knoch, D. (2015). A neural trait approach to exploring individual differences in social preferences. Frontiers in Behavioral Neuroscience, 8, 458.

Nelson, D. W., Klein, C. T., \& Irvin, J. E. (2003). Motivational antecedents of empathy: Inhibiting effects of fatigue. Basic and Applied Social Psychology, 25(1), 37-50.

Newman, M. E. (2006). Finding community structure in networks using the eigenvectors of matrices. Physical Review E, 74(3), 036104.

Newman, M. E., \& Girvan, M. (2004). Finding and evaluating community structure in networks. Physical Review E, 69(2), 026113.

Power, J. D., Mitra, A., Laumann, T. O., Snyder, A. Z., Schlaggar, B. L., \& Petersen, S. E. (2014). Methods to detect, characterize, and remove motion artifact in resting state fMRI. NeuroImage, 84 , 320-341.

Richter, L., \& Kruglanski, A. W. (1999). Motivated search for common ground: Need for closure effects on audience design in interpersonal communication. Personality and Social Psychology Bulletin, 25(9), 1101-1114.

Roets, A., \& Hiel, A. (2008). Why some hate to dilly-dally and others do not: The arousal-invoking capacity of decision-making for lowand high-scoring need for closure individuals. Social Cognition, 26(3), 333-346.

Roets, A., Kruglanski, A. W., Kossowska, M., Pierro, A., \& Hong, Y. (2015). The motivated gatekeeper of our minds: New directions in need for closure theory and research. Advances in experimental social psychology (52 vol., pp. 221-283). Elsevier

Rubinov, M., \& Sporns, O. (2010). Complex network measures of brain connectivity: uses and interpretations. NeuroImage, 52(3), 1059-1069.

Schrouff, J., Rosa, M. J., Rondina, J. M., Marquand, A. F., Chu, C., Ashburner, J., \& Mourao-Miranda, J. (2013). PRoNTo: pattern recognition for neuroimaging toolbox. Neuroinformatics, 11(3), 319-337.

Schumpe, B. M., Brizi, A., Giacomantonio, M., Panno, A., Kopetz, C., Kosta, M., \& Mannetti, L. (2017). Need for Cognitive Closure 
decreases risk taking and motivates discounting of delayed rewards. Personality and Individual Differences, 107, 66-71.

Senderecka, M., Szewczyk, J., Wichary, S., \& Kossowska, M. (2018). Individual differences in decisiveness: ERP correlates of response inhibition and error monitoring. Psychophysiology, 55(10), e13198

Shiloh, S., Koren, S., \& Zakay, D. (2001). Individual differences in compensatory decision-making style and need for closure as correlates of subjective decision complexity and difficulty. Personality and Individual Differences, 30(4), 699-710.

Sparkman, D. J., \& Blanchar, J. C. (2017). Examining relationships among epistemic motivation, perspective taking, and prejudice: A test of two explanatory models. Personality and Individual Differences, 114, 48-56.

Starcevic, V., \& Berle, D. (2006). Cognitive specificity of anxiety disorders: a review of selected key constructs. Depression and Anxiety, 23(2), 51-61.

Stark, J. H., \& Milyavsky, M. (2019). Towards a better understanding of lawyers' judgmental biases in client representation: the role of need for cognitive closure. Washington University Journal of Law \& Policy, 59, 173.

Szumowska, E., \& Kossowska, M. (2016). Need for closure and multitasking performance: The role of shifting ability. Personality and Individual Differences, 96, 12-17.

Szumowska, E., \& Kossowska, M. (2017). Need for cognitive closure and attention allocation during multitasking: Evidence from eye-tracking studies. Personality and Individual Differences, 111, 272-280.

Szumowska, E., Popławska-Boruc, A., \& Kossowska, M. (2018). How many things do you (like to) do at once? The relationship between need for closure and multitasking preference and behavior. Personality and Individual Differences, 134, 222-231.

Thompson-Schill, S. L., Jonides, J., Marshuetz, C., Smith, E. E., D'Esposito, M., Kan, I. P., \& Swick, D. (2002). Effects of frontal lobe damage on interference effects in working memory. Cognitive, Affective, \& Behavioral Neuroscience, 2(2), 109-120.

Tipping, M. E. (2001). Sparse Bayesian learning and the relevance vector machine. Journal of Machine Learning Research, I(Jun), 211-244.

Todor, I. (2014). Need for closure and cognitive inhibition of unwanted or irrelevant information. Procedia - Social and Behavioral Sciences, 141, 712-717. https://doi.org/10.1016/j.sbspro.2014.05. 125 .

Tzourio-Mazoyer, N., Landeau, B., Papathanassiou, D., Crivello, F., Etard, O., Delcroix, N., \& Joliot, M. (2002). Automated anatomical labeling of activations in SPM using a macroscopic anatomical parcellation of the MNI MRI single-subject brain. NeuroImage, 15(1), 273-289.

Varoquaux, G., Raamana, P. R., Engemann, D. A., Hoyos-Idrobo, A., Schwartz, Y., \& Thirion, B. (2017). Assessing and tuning brain decoders: cross-validation, caveats, and guidelines. NeuroImage, $145,166-179$.

Viola, V., Tosoni, A., Brizi, A., Salvato, I., Kruglanski, A. W., Galati, G., \& Mannetti, L. (2015). Need for cognitive closure modulates how perceptual decisions are affected by task difficulty and outcome relevance. PLoS ONE, 10(12), e0146002
Viola, V., Tosoni, A., Kruglanski, A. W., Galati, G., \& Mannetti, L. (2014). Routes of motivation: stable psychological dispositions are associated with dynamic changes in cortico-cortical functional connectivity. PLoS One1, 9(6), e98010. https://doi.org/10.1371/ journal.pone.0098010.

Wang, J., Wang, X., Xia, M., Liao, X., Evans, A., \& He, Y. (2015). GRETNA: a graph theoretical network analysis toolbox for imaging connectomics. Frontiers in Human Neuroscience, 9, 386.

Webber, D., Babush, M., Schori-Eyal, N., Vazeou-Nieuwenhuis, A., Hettiarachchi, M., Bélanger, J. J., \& Kruglanski, A. W. (2018). The road to extremism: Field and experimental evidence that significance loss-induced need for closure fosters radicalization. Journal of Personality and Social Psychology, 114(2), 270.

Webster, D. M., \& Kruglanski, A. W. (1994). Individual differences in need for cognitive closure. Journal of Personality and Social Psychology, 67(6), 1049.

Whitwell, J. L. (2009). Voxel-based morphometry: an automated technique for assessing structural changes in the brain. Journal of Neuroscience, 29(31), 9661-9664.

Wronska, M. K., Bujacz, A., Gocłowska, M. A., Rietzschel, E. F., \& Nijstad, B. A. (2019). Person-task fit: Emotional consequences of performing divergent versus convergent thinking tasks depend on need for cognitive closure. Personality and Individual Differences, 142, 172-178.

Wu, B., Chen, L., Hu, Z., Zhang, W., \& Liang, J. (2008). A relevance vector regression based metamodeling approach for complex system analysis. Paper presented at the 2008 Asia Simulation Conference-7th International Conference on System Simulation and Scientific Computing.

Xu, Y., Lin, Q., Han, Z., He, Y., \& Bi, Y. (2016). Intrinsic functional network architecture of human semantic processing: modules and hubs. NeuroImage, 132, 542-555.

Yan, C., Wang, X. D., Zuo, X. N., \& Zang, Y. F. (2016). DPABI: data processing \& analysis for (resting-state) brain imaging. Neuroinformatics, 14(3), 339-351.

Yan, C., \& Zang, Y. (2010). DPARSF: a MATLAB toolbox for" pipeline" data analysis of resting-state fMRI. Frontiers in Systems Neuroscience, 4, 13.

Yan, C. G., Cheung, B., Kelly, C., Colcombe, S., Craddock, R. C., Di Martino, A., \& Milham, M. P. (2013). A comprehensive assessment of regional variation in the impact of head micromovements on functional connectomics. NeuroImage, 76, 183-201.

Yarkoni, T., Poldrack, R. A., Nichols, T. E., Van Essen, D. C., \& Wager, T. D. (2011). Large-scale automated synthesis of human functional neuroimaging data. Nature Methods, 8(8), 665-670.

Yeo, B. T., Krienen, F. M., Sepulcre, J., Sabuncu, M. R., Lashkari, D., Hollinshead, M., \& Polimeni, J. R. (2011). The organization of the human cerebral cortex estimated by intrinsic functional connectivity. Journal of Neurophysiology, 106(3), 1125-1165.

Zhang, R., Geng, X., \& Lee, T. M. (2017). Large-scale functional neural network correlates of response inhibition: an fMRI metaanalysis. Brain Structure and Function, 222(9), 3973-3990.

Zuo, X. N., Xu, T., \& Milham, M. P. (2019). Harnessing reliability for neuroscience research. Nature Human Behaviour, 3(8), 768-771.

Publisher's Note Springer Nature remains neutral with regard to jurisdictional claims in published maps and institutional affiliations. 